\title{
Instrumento terapéutico para tratamiento de la tenosinovitis De Quervain
}

\section{A therapeutic instrument for the treatment of De Quervain's Tenosynovitis}

\author{
Michelle Copo-Torres ${ }^{1 a}$, Francisco Echeverría-Tamayo ${ }^{\text {1b }}$, Santiago Santamaría-Bedón ${ }^{1 c}$, \\ Pablo Amancha-Proaño 1d \\ ${ }^{1}$ Escuela de Diseño Industrial, Universidad Católica del Ecuador, Ambato, Ecuador. Correos electrónicos: \\ a evelyn.m.copo.t@ pucesa.edu.ec, ${ }^{\mathrm{b}}$ fecheverria@ pucesa.edu.ec, ${ }^{\mathrm{c}}$ ssantamaría@ pucesa.edu.ec, \\ d pamancha@ pucesa.edu.ec. Orcid: a 0000-0002-6495-3471, b 0000-0002-3364-7260, c 0000-0003-2593-6692, \\ ${ }^{d} 0000-0003-1502-6118$
}

Recibido: 26 octubre, 2020. Aceptado: 10 marzo, 2021. Versión final: 9 junio, 2021.

\begin{abstract}
Resumen
La tenosinovitis De Quervain se define como una estenosis de los tendones del abductor largo y extensor corto del pulgar, lo cual reduce la fuerza y destreza de la mano. Se origina debido a un accidente laboral o una lesión existente. El trabajo tiene como objetivo diseñar un instrumento terapéutico para el tratamiento de esta patología. La metodología de diseño que se aplica está dividida en cuatro fases: planificación y aclaración de la tarea, diseño conceptual, realización y detallado. Como resultado se obtiene un instrumento autoasistido de apoyo en la terapia física, que aplica el programa Tendon Gliding Exercise (TGE) para determinar los ejercicios terapéuticos del instrumento. Este posee dispositivos intercambiables para mano y muñeca, que le permiten al paciente realizar movimientos ideales de flexión y extensión de los dedos.
\end{abstract}

Palabras clave: De Quervain; metodología de diseño; TGE; ejercicios terapéuticos; apoyo en la terapia física, ejercicio de deslizamiento de tendones.

\begin{abstract}
De Quervain's tenosynovitis is defined as stenosis of the abductor pollicis longus tendons and extensor pollicis brevis that reduces the strength and dexterity of the hand. It is the result of a work accident or an existing injury. The research objective is to design a therapeutic instrument for a pathology treatment. The design methodology applied is divided into 4 phases: planning and clarification of the task, conceptual design, realization design, and detailed design. As a result, there is self-assisted physical therapy support, which uses the Tendon Gliding Exercise (TGE) program to determine the therapeutic exercises of the instrument. It has interchangeable hand and wrist devices, that allow the patient to perform ideal flexion and extension movements of the fingers.
\end{abstract}

Keywords: De Quervain's; design methodology; TGE; therapeutic exercises; Tendon Gliding Exercise; physical therapy support.

\section{Introducción}

Los trastornos musculoesqueléticos (TME) son lesiones presentes en músculos, tendones, articulaciones y nervios, y producen molestias, dolor localizado y restricción de la movilidad [1], [2]. Pese a que los TME tienen mayor influencia en personas mayores, en 2017, entre el $20 \%$ y el $33 \%$ de la población mundial mostró esta enfermedad, siendo esta la segunda causa de discapacidad [3], [4].

ISSN impreso: 1657 - 4583. ISSN en línea: 2145 - 8456, CC BY-ND 4.0@ @() 요

Como citar: M. Copo-Torres, F. Echeverría-Tamayo, S. Santamaría-Bedón, P. Amancha-Proaño, "Instrumento terapéutico para tratamiento de la tenosinovitis De Quervain," Rev. UIS Ing., vol. 20, no. 4, pp. 1-12, 2021, doi: 10.18273/revuin.v20n4-2021001. 
Estudios han identificado varios factores de riesgo físico en actividades laborales que requieren esfuerzos excesivos, tareas altamente repetitivas, adopción de posturas no neutrales y exposición a vibraciones que generan TME, con una afectación mayor en cuello, extremidades superiores y espalda [5]. Van Middelkoop et al. [6] describen que los TME específicos de la mano son dedo en gatillo, fenómeno de Raynaud, enfermedad de Dupuytren y la tenosinovitis De Quervain.

La tenosinovitis De Quervain se define como una estenosis de los tendones del abductor largo y extensor corto del pulgar, lo cual reduce la fuerza y destreza de la mano [7], [8]. Esta se origina por un accidente laboral o por una lesión previamente existente que se agrava por la actividad física, con una incidencia del $0,5 \%$ entre los hombres y del $1,3 \%$ entre las mujeres [1], [9]. El tratamiento no quirúrgico, que incluye reposo articular asociado con antiinflamatorios o infiltración local de corticoides, proporciona un alivio eficaz de los síntomas en los pacientes; en casos resistentes, se recomienda la liberación quirúrgica del primer compartimento dorsal [9]. Regularmente, la fisioterapia suele reservarse para los fracasos del tratamiento quirúrgico, aunque en ocasiones se tiene la posibilidad de intervención temprana [8].

\subsection{Ejercicios para el deslizamiento del tendón}

El rol del ejercicio terapéutico en el tratamiento del dolor ha sido objeto de especial atención a lo largo de la historia de la medicina [10]. Ejercicios de movimiento en la mano generan disminución de dolor, debido a la insensibilización de las vías de trasmisión periféricas, lo que aumenta la resistencia tendinosa e interrumpe el flujo sanguíneo capilar reduciendo el proceso inflamatorio [11]. Estos movimientos son considerados dentro de programas terapéuticos de rehabilitación (véase tabla 1), los cuales combinan determinados ejercicios para mejoras en las articulaciones afectadas en relación con el movimiento obtenido y el fortalecimiento de los grupos musculares más relevantes para la función manual [12].

\subsection{Ejercicios para el deslizamiento del tendón}

Los ejercicios para el deslizamiento del tendón (TGE, por sus siglas en inglés) tienen por objetivo el deslizamiento de los tendones flexores mediante el movimiento del pulgar y tres posiciones básicas del puño.

Este tipo de ejercicios ayuda a recuperar la movilidad y musculatura perdida a causa del dolor producido por movimientos forzosos del pulgar, y está dentro del rango de movimientos (véase tabla 2) para un correcto deslizamiento de los tendones [13].

Tabla 2. Rango de movimientos de una persona sana

\begin{tabular}{|l|c|}
\hline \multicolumn{1}{|c|}{ Movimiento } & $\begin{array}{c}\text { Rango de movimiento } \\
\text { persona sana }\end{array}$ \\
\hline Flexión & $90^{\circ}$ \\
\hline Extensión & $80^{\circ}$ \\
\hline Derivación radial & $15^{\circ}$ \\
\hline Desviación cubital & $30^{\circ}-45^{\circ}$ \\
\hline Pronación & $85^{\circ}$ \\
\hline Supinación & $90^{\circ}$ \\
\hline
\end{tabular}

Fuente: [13].

Tabla 1. Tipos de programas de rehabilitación

\begin{tabular}{|c|c|c|}
\hline Programa & Descripción & $\begin{array}{l}\text { Ejercicio } \\
\end{array}$ \\
\hline Movilidad & $\begin{array}{l}\text { Movimiento articular de la } \\
\text { mano para mejorar el } \\
\text { recorrido articular. }\end{array}$ & $\begin{array}{l}\text { - Oposición del pulgar } \\
\text { - Extensión individual de cada dedo } \\
\text { - Flexión y extensión de metacarpofalángicas } \\
\text { - Flexión y extensión de interfalángicas proximales } \\
\text { - Flexión y extensión de interfalángicas distales. }\end{array}$ \\
\hline Fortalecimiento & Fortalecimiento muscular. & $\begin{array}{l}\text { - Sujetar un papel entre los dedos } \\
\text { - Desviaciones radiales resistidas con plastilina } \\
\text { - Abducción y aducción de dedos resistida con plastilina. }\end{array}$ \\
\hline Combinado & $\begin{array}{l}\text { Movilidad y fortalecimiento } \\
\text { de mano y muñeca, y puede } \\
\text { ser asistido por material } \\
\text { didáctico. }\end{array}$ & $\begin{array}{l}\text { - Hacer un puño } \\
\text { - Extensión individual de cada dedo } \\
\text { - Oposición del pulgar } \\
\text { - Sujetar un papel entre los dedos } \\
\text { - Desviaciones radiales resistidas con plastilina } \\
\text { - Abducción y aducción de dedos resistida con plastilina. }\end{array}$ \\
\hline
\end{tabular}

Fuente: elaboración propia. 


\section{Metodología}

El proceso metodológico de diseño se divide en cuatro fases [14]: planificación y aclaración de la tarea, diseño conceptual, diseño de realización y diseño detallado (véase figura 1). Las acciones preliminares recopilan la información sobre requerimientos del usuario para concluir con las especificaciones de diseño; seguido por el desarrollo de estructuras funcionales y la selección del concepto principal. A partir del concepto seleccionado se produce el diseño definitivo en concordancia con las especificaciones técnicas, las cuales están basadas en dimensiones funcionales, ergonómicas y formales. Finalmente, el diseño de detalle establecerá varias especificaciones del prototipo como la geometría, materiales y sistemas de ensamble, para concluir con su construcción mediante tecnologías de fabricación rápida.

\subsection{Planificación y aclaración de la tarea}

La palabra 'necesidad' marca cualquier atributo de un potencial producto deseado por un usuario. Se busca como resultado una lista jerárquica de enunciados que se convertirán en objetivos centrales a efectuar [15]. Para que las necesidades del usuario puedan cumplirse, el instrumento debe presentar una jerarquía, que inicia con la funcionalidad, seguido de la usabilidad y, por último, la parte formal o estética del producto.

En el aspecto funcional, el instrumento debe permitir la ejecución de los movimientos establecidos por ejercicios terapéuticos. La usabilidad marca las necesidades ergonómicas, la interacción del usuario con el producto, la facilidad y practicidad de uso, la adaptabilidad mediante la consideración de la antropometría de la mano y la necesidad de que sus características funcionales se comuniquen de manera eficaz. El aspecto formal considera los caracteres estéticos del instrumento: que exprese coherencia y un equilibrio a través de la geometría.

A continuación, se determinan las especificaciones del producto a partir de las necesidades identificadas, las cuales permiten generar un buen concepto de diseño y denotan las características ingenieriles y formales que el instrumento terapéutico debe contemplar. Para ello, se establece una matriz de relación entre las necesidades del usuario con las características técnicas del producto para identificar las especificaciones de diseño.

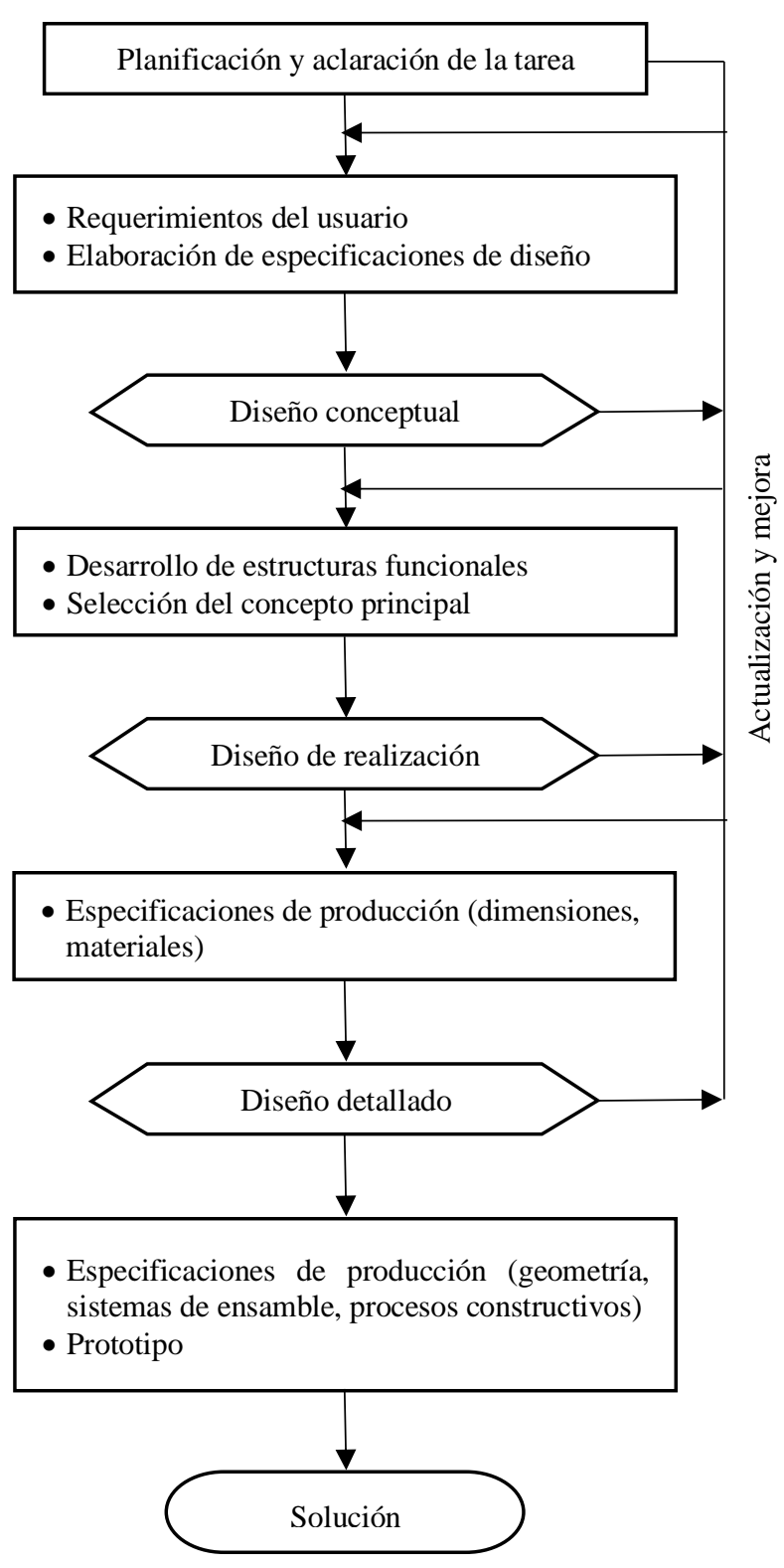

Figura 1. Pasos del proceso de planificación y diseño. Fuente: elaboración propia.

\subsection{Diseño conceptual}

Mediante el establecimiento y combinación de estructuras funcionales, se establece el diseño conceptual para generar la solución [14]. Como recurso para comunicar y materializar las especificaciones técnicas se emplean bocetos y modelos de estudio. En esta etapa, altamente creativa, el boceto presenta ventajas para la generación gradual de variantes en el diseño y los componentes. 


\subsection{Diseño de realización}

A partir del concepto del producto se desarrolla el diseño, de acuerdo con los criterios ingenieriles y formales definidos. El diseño de realización establece la geometría a partir de la antropometría de la mano, y especifica los materiales para la construcción de un prototipo funcional.

\subsection{Diseño detallado}

Se genera un modelado 3D CAD y los planos dimensionales para establecer los sistemas de ensamble de cada componente. La información que suministra un prototipo puede acoplarse a cualquier punto del proceso de diseño y no se ajusta a ningún espacio en específico [14]. Por lo tanto, en el diseño detallado, se desarrolla el prototipo del instrumento terapéutico en una versión a escala real y operacional, que permita demostrar el cumplimiento de las especificaciones del producto, su funcionalidad y usabilidad. Como tecnología de fabricación rápida, se emplea el modelado por deposición fundida (FDM, por sus siglas en inglés), que consiste en una manufactura aditiva donde plástico fundido se extruye a través de una boquilla y se deposita en capas [16].

\section{Resultados}

\subsection{Planeación: necesidades y especificaciones}

Los datos recolectados de entrevistas a especialistas y fichas de observación a pacientes son sintetizados en un cuadro de necesidades de uso, función, forma, estructura, nivel social y técnico productivas (véase tabla 3 ).
Posteriormente, estas necesidades son analizadas para valorar su jerarquía, la cual inicia con la funcionalidad, seguida de la usabilidad y, por último, la parte formal o estética del producto (véase tabla 4).

Se elabora la matriz de relación entre las necesidades del usuario y las características técnicas del producto, con lo cual se determina las especificaciones medibles de ingeniería en el diseño (véase tabla 5).

Esta matriz permite generar las dimensiones fundamentales del prototipo, que contempla el concepto del producto mediante una descripción aproximada de su funcionalidad, usabilidad y forma.

Tabla 4. Lista jerárquica de necesidades

\begin{tabular}{|cl|}
\hline \multicolumn{2}{|c|}{ Necesidades } \\
\hline 1. & Movimientos de flexión, extensión de los dedos \\
\hline 2. & Movimientos de oposición del pulgar \\
\hline 3. & Compresión en muñeca \\
\hline 4. & Estimulación en yema de dedos \\
\hline 5. & Formas que se adapten a la anatomía de la mano \\
\hline 6. & Permita realizar cambios de ejercicios \\
\hline 7. & Uso terapéutico \\
\hline 8. & Instrumento autoasistido \\
\hline 9. & Fácil de colocar para uso cotidiano \\
\hline 10. & Cómodo para sujetarlo \\
\hline 11. & Comprensión en su uso mediante la forma \\
\hline 12. & Motivación en la realización de terapias \\
\hline
\end{tabular}

Fuente: elaboración propia.

Tabla 3. Lista de necesidades del instrumento terapéutico

\begin{tabular}{|c|l|}
\hline \multicolumn{1}{|c|}{ Uso } & $\begin{array}{l}\text { Antropométrico } \\
\text { Ergonómico } \\
\text { Instrumento autoasistido }\end{array}$ \\
\hline \multirow{3}{*}{ Funcionales } & $\begin{array}{l}\text { Alivio inmediato } \\
\text { Movimientos para ejercitar, estimular los dedos de la mano } \\
\\
\text { Versátil } \\
\text { Compresión para el miembro superior }\end{array}$ \\
\hline \multirow{2}{*}{ Formales } & $\begin{array}{l}\text { Estético } \\
\text { Anatómico a la mano }\end{array}$ \\
\hline \multirow{2}{*}{ Estructurales } & $\begin{array}{l}\text { Liviano (densidad baja) } \\
\text { Funciones específicas para sus componentes }\end{array}$ \\
\hline Social & Motivación al desarrollar terapias \\
\hline \multirow{2}{*}{ Técnico productivas } & $\begin{array}{l}\text { Sustentabilidad, sostenibilidad económica } \\
\text { Materiales comunes } \\
\text { Maquinaria existente en el país y que cumpla con normas y estándares de fabricación }\end{array}$ \\
\hline
\end{tabular}

Fuente: elaboración propia. 
Tabla 5. Matriz de especificaciones del instrumento terapéutico basados en sus necesidades

\begin{tabular}{|c|c|c|c|c|c|c|c|c|c|}
\hline Necesidades & 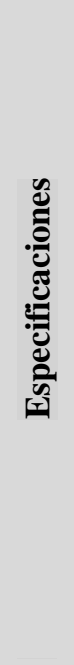 & 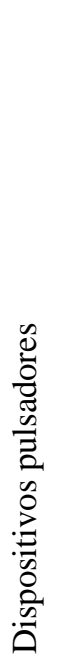 & 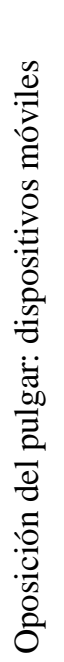 & 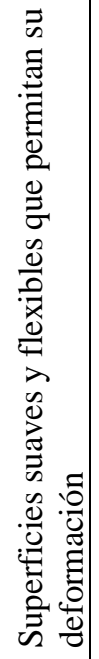 & 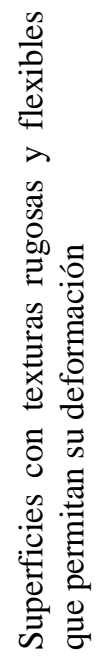 & 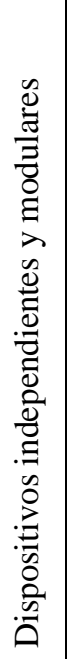 & 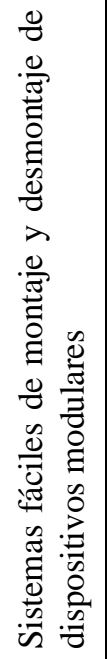 & 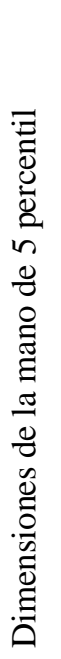 & 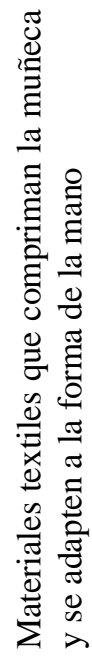 \\
\hline 1. Movimientos de flexión, extensión de los dedo & & • & - & & & • & - & & \\
\hline 2. Movimientos de oposición del pulgar & & & • & & & • & - & & \\
\hline 3. Compresión en muñeca & & & & & & & & & - \\
\hline 4. Estimulación en yema de dedos & & & & - & & $\bullet$ & - & & \\
\hline 5. Formas que se adapten a la anatomía de la man & & & - & & & & & - & \\
\hline 6. Permita realizar cambios de ejercicios & & & - & & & - & - & & \\
\hline 7. Uso terapéutico & & - & $\cdot$ & - & - & & & & - \\
\hline 8. Instrumento autoasistido & & & - & & & - & - & & \\
\hline 9. Fácil de colocar para uso cotidiano & & & - & & & - & - & & \\
\hline 10. Cómodo para sujetarlo & & • & - & & & - & - & & \\
\hline 11. Comprensión en su uso mediante la forma & & • & & • & • & • & • & • & - \\
\hline 12. Motivación en la realización de terapias & & - & - & & & & - & & \\
\hline
\end{tabular}

Fuente: elaboración propia.

Bajo esta primicia, el instrumento terapéutico para tratamiento de tenosinovitis De Quervain describe las siguientes cualidades:

1. Instrumento autoasistido de apoyo en la terapia física de personas que sufren dolores en la mano y muñeca a causa de la tenosinovitis De Quervain.

2. Motiva los movimientos de flexión y extensión de los dedos a través de dispositivos independientes y modulares, los cuales podrán intercambiarse para realizar los diferentes ejercicios de deslizamiento de tendones, así:

- Mano recta: guante de materiales textiles que compriman la muñeca.

- Puño de gancho: a través de dispositivos pulsadores.
- Puño completo: componentes con superficies suaves y flexibles que permitan su deformación al comprimirlos con los dedos.

- Puño recto: componentes con superficies rugosas y flexibles que permitan su deformación al comprimirlos con los dedos.

- Oposición del pulgar: movimiento pendular del pulgar mediante dispositivo móvil.

Se aplica el programa Tendon Gliding Exercise (TGE) para determinar los ejercicios terapéuticos del instrumento (véase figura 2), el cual consiste en una serie de movimientos, donde los tendones flexores del pulgar y las cuatro posiciones básicas de puño se deslizan a su máximo potencial [17].

Adicionalmente, para establecer los parámetros de diseño, se dispuso la biomecánica de la mano, en especial de la muñeca (véase figura 3), para determinar sus movimientos funcionales. 


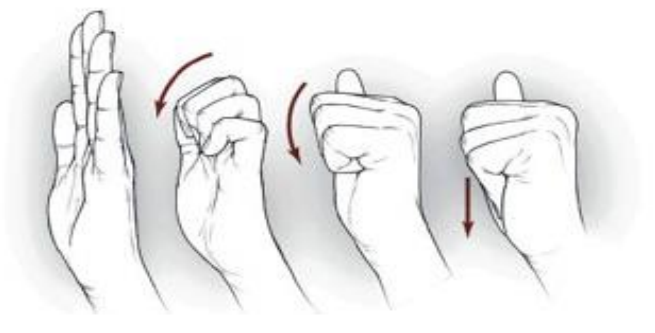

Figura 2. Tendon Gliding Exercise. Fuente:[17].

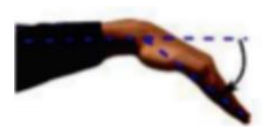

Flexión

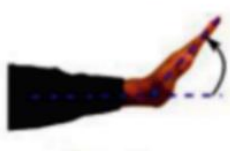

Extensión

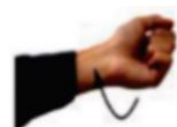

Supinación

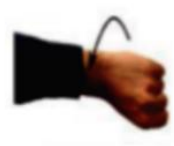

Pronación

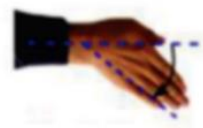

Desviación
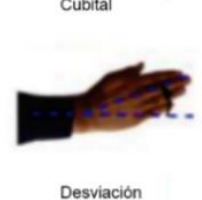

Desviación
Radial
Figura 3. Movimientos de la muñeca. Fuente: [13].

\subsection{Bocetos y modelos de estudio}

El boceto es la principal herramienta de investigación y desarrollo que el diseño conceptual emplea. Aquí, se exploran y evalúan de manera visual las primeras soluciones a través de principios de dibujo que simulan la forma física del producto, sus características funcionales y formales. El instrumento consta de un guante versátil y ajustable (véase figura 4), que funciona como inmovilizador para mantener la postura correcta de mano/muñeca y evitar los movimientos inapropiados.
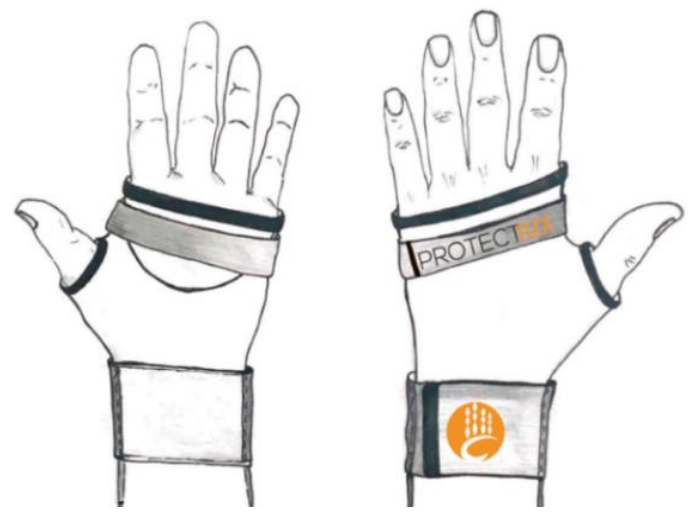

Figura 4. Boceto de guante inmovilizador. Fuente: elaboración propia.

A este se adhieren, mediante correas, tres dispositivos que funcionan de manera independiente, los cuales se detallan a continuación:
1. Dispositivo de relajación palmar: destinado a la relajación de la mano y muñeca, mediante terapia de liberación de tensión en articulaciones y tendones. Funciona mediante la presión que ejercen los dedos sobre una superficie flexible y rugosa. Su forma se adapta a la anatomía de la mano (véase figura 5).
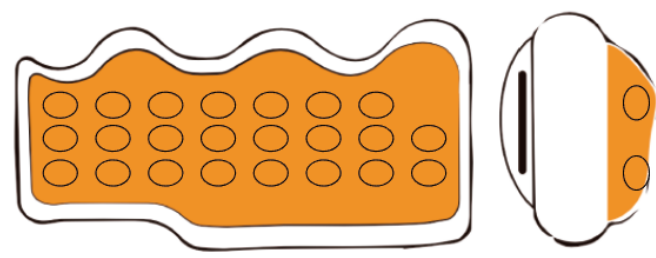

Figura 5. Boceto de dispositivo de relajación palmar. Fuente: elaboración propia.

2. Dispositivo de motricidad palmar: permite recuperar la movilidad de los dedos y motricidad de la mano. $\mathrm{Su}$ accionamiento es mediante el movimiento de flexión, que incentiva a los dedos índice, medio y anular al desarrollo de ejercicios de manera individual. Cuenta con pulsadores que se conectan a luces LED para su interacción (véase figura 6).

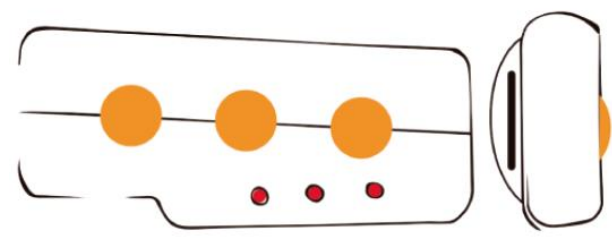

Figura 6. Boceto de dispositivo de motricidad palmar. Fuente: elaboración propia.

3. Dispositivo de movilidad pulgar: su forma adaptable a la anatomía de la mano crea un movimiento de oposición del pulgar, lo cual ayuda a la regeneración de los tendones. Funciona mediante la colocación del dedo pulgar sobre un pasador deslizante (véase figura 7).

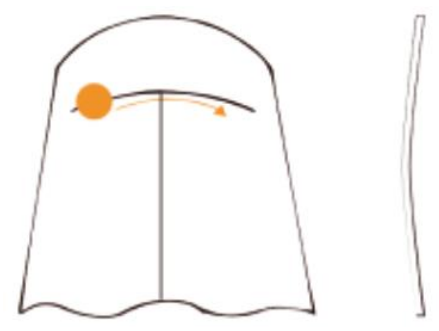

Figura 7. Boceto de dispositivo de movilidad pulgar. Fuente: elaboración propia. 
Para obtener una apreciación tridimensional del modelo propuesto, se desarrollan maquetas para la observación y validación de aspectos relevantes al instrumento, que permiten obtener un proceso iterativo de aprendizaje. Para ello, se experimentó con materiales moldeables e impresiones 3D de baja calidad, hasta conseguir la forma y dimensiones apropiadas que se adaptan a la mano y a sus movimientos (véase figura 8).

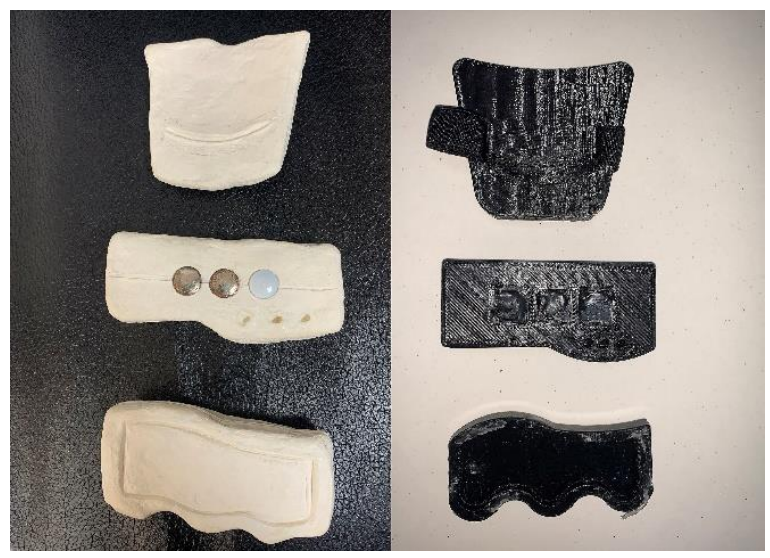

Figura 8. Modelos de estudio de los instrumentos terapéuticos. Fuente: elaboración propia.

\subsection{Dimensiones}

Se establecen las dimensiones funcionales del instrumento mediante la aplicación de las medidas antropométricas de la mano, comprendidas en los valores de los percentiles 50-95 (véase tabla 6), así como de la experiencia obtenida con los modelos de estudio de la fase de diseño conceptual. La tabla 7 indica las dimensiones generales de los distintos componentes del instrumento terapéutico.

Tabla 6. Dimensiones antropométricas de la mano

\begin{tabular}{|l|c|}
\hline Percentil 95 & Dimensiones (mm) \\
\hline Largo de la mano I & 205 \\
\hline Largo de palma J & 118 \\
\hline Ancho de mano K & 96 \\
\hline Percentil 50 & Dimensiones (mm) \\
\hline Largo de la mano I & 178 \\
\hline Largo de palma J & 100 \\
\hline Ancho de mano K & 82 \\
\hline
\end{tabular}

Fuente: [18].

\subsection{Materiales}

Se estableció un análisis comparativo para la selección del material de los componentes, tomando como base su aplicación, módulo de Young y densidad.

El instrumento terapéutico se divide en dos partes: un guante y tres aplicaciones, los mismos que emplean los siguientes materiales:

1. Neopreno (policloropreno): es empleado para el guante debido a su baja transmisión térmica, durabilidad y excelentes propiedades (véase tabla 8). $\mathrm{Su}$ proceso de manufactura se desarrolla mediante el patronaje, corte y armado de cada una de las piezas.

2. ABS (acrilonitrilo butadieno estireno): se usa para los tres componentes del instrumento (véase tabla 9). Este material es procesado mediante inyección en moldes o impresión 3D.

Tabla 7. Dimensiones generales del instrumento terapéutico

\begin{tabular}{|l|c|}
\hline \multicolumn{1}{|c|}{ Componentes } & Dimensiones (mm) \\
\hline Guante inmovilizador & $195 \times 100 \times 50$ \\
\hline $\begin{array}{l}\text { Dispositivo de } \\
\text { relajación palmar }\end{array}$ & $82 \times 36 \times 10$ \\
\hline $\begin{array}{l}\text { Dispositivo de } \\
\text { motricidad palmar }\end{array}$ & $85 \times 36 \times 16$ \\
\hline $\begin{array}{l}\text { Dispositivo de } \\
\text { movilidad pulgar }\end{array}$ & $81 \times 62 \times 8$ \\
\hline
\end{tabular}

Fuente: elaboración propia.

\subsection{Prototipo}

Dentro de la etapa de diseño de detalle se define la geometría e interrelación de los subsistemas y componentes que conforman el producto, mediante el modelado 3D CAD que permita su construcción. Se presentan modelos virtuales y prototipos que validan sus diferentes características, como: desempeño funcional, dimensiones, aspectos de uso, apariencia, sistemas de ensamble, entre otras.

Los componentes de los dispositivos están conformados por ABS y ensamblados mediante conectores magnéticos. Los esquemas de unión se encuentran ilustrados en las figuras 10, 12, 14 y 15. Los planos dimensionales se encuentran representados en las figuras 9, 11 y 13. La figura 16 muestra el prototipo real en uso con sus distintos complementos. 
Tabla 8. Propiedades del neopreno

\begin{tabular}{|c|c|c|}
\hline \multicolumn{3}{|c|}{$\begin{array}{c}\text { Policloropreno (CR, 17-50 \% carbón negro) } \\
\text { Neopreno }\end{array}$} \\
\hline Descripción & Valor & Unidad \\
\hline \multicolumn{3}{|l|}{ Propiedades mecánicas } \\
\hline Módulo de Young & $\begin{array}{c}0,0021- \\
0,015\end{array}$ & $\mathrm{GPa}$ \\
\hline Límite elástico & $10,5-21,3$ & $\mathrm{MPa}$ \\
\hline Fuerza de tensión & $11,4-21,3$ & $\mathrm{MPa}$ \\
\hline Elongación & $120-780$ & $\%$ presión \\
\hline Módulo de compresión & $\begin{array}{c}0,0021- \\
0,015\end{array}$ & $\mathrm{GPa}$ \\
\hline Fuerza compresiva & $12,6-25,6$ & $\mathrm{MPa}$ \\
\hline Módulo de flexión & $\begin{array}{c}0,0021- \\
0,015\end{array}$ & $\mathrm{GPa}$ \\
\hline Dureza & $4-7$ & $\mathrm{HV}$ \\
\hline \multicolumn{3}{|l|}{ Propiedades térmicas } \\
\hline Conductividad térmica & $0,2-0,9$ & $\mathrm{~W} / \mathrm{m}^{\circ} \mathrm{C}$ \\
\hline \multicolumn{3}{|l|}{ Propiedades eléctricas } \\
\hline Conductividad eléctrica & $\begin{array}{c}1,72 \mathrm{e}-10- \\
1,72 \mathrm{e}-4 \\
\end{array}$ & $\%$ IACS \\
\hline \multicolumn{3}{|c|}{ Absorción y permeabilidad } \\
\hline $\begin{array}{l}\text { Absorción de agua } \\
\text { ( } 24 \text { horas) }\end{array}$ & $0,6-0,8$ & $\%$ \\
\hline \multicolumn{3}{|l|}{ Durabilidad } \\
\hline Agua (dulce) & & Excelente \\
\hline Agua (salada) & & Excelente \\
\hline Ácidos débiles & & Aceptable \\
\hline Alcalinos fuertes & & Excelente \\
\hline Ácidos fuertes & & Uso limitado \\
\hline Radiación UV & & Bueno \\
\hline
\end{tabular}

Fuente: [19].
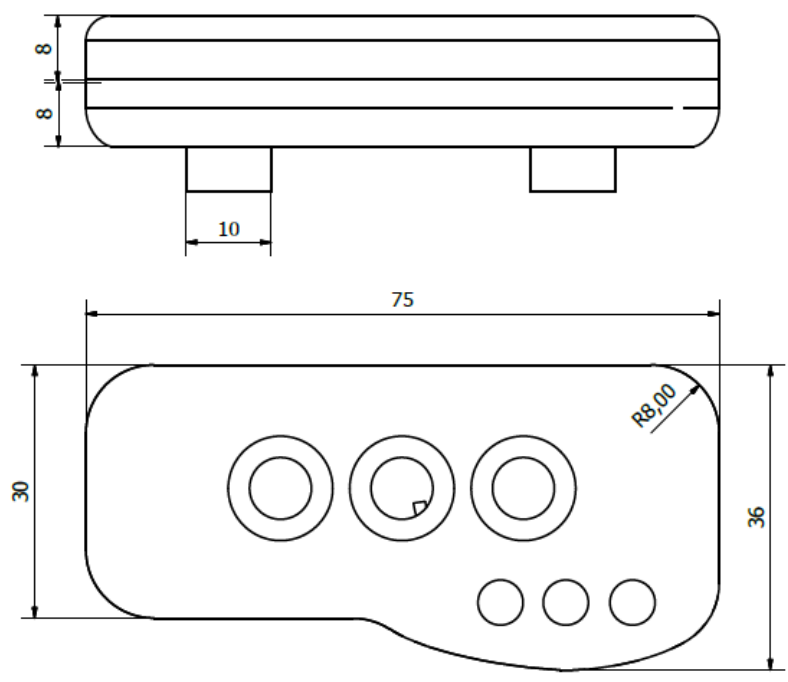

Figura 9. Planos dimensionales del dispositivo de motricidad palmar. Fuente: elaboración propia.

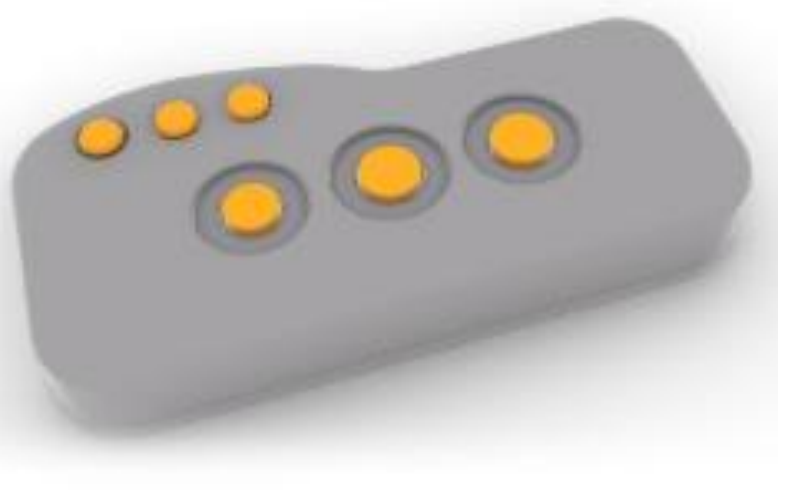

Figura 10. Modelo virtual del dispositivo de motricidad palmar. Fuente: elaboración propia.

Tabla 9. Propiedades del ABS

\begin{tabular}{|c|c|c|}
\hline \multicolumn{3}{|c|}{ ABS (moldeo por inyección de alto impacto) } \\
\hline Descripción & Valor & Unidad \\
\hline \multicolumn{3}{|l|}{ Propiedades mecánicas } \\
\hline Módulo de Young & $1,1-2,41$ & $\mathrm{GPa}$ \\
\hline Límite elástico & $18,5-40,7$ & $\mathrm{MPa}$ \\
\hline Fuerza de tensión & $30,3-43,4$ & $\mathrm{MPa}$ \\
\hline Elongación & $5-75$ & \% presión \\
\hline Módulo de compresión & $\begin{array}{c}0,0021- \\
0,015 \\
\end{array}$ & $\mathrm{GPa}$ \\
\hline Fuerza compresiva & $0,963-2,06$ & $\mathrm{MPa}$ \\
\hline Módulo de flexión & $1,23-2,58$ & $\mathrm{GPa}$ \\
\hline Dureza & $6-12$ & $\mathrm{HV}$ \\
\hline \multicolumn{3}{|l|}{ Propiedades térmicas } \\
\hline Conductividad térmica & $0,19-0,21$ & $\mathrm{~W} / \mathrm{m}^{\circ} \mathrm{C}$ \\
\hline \multicolumn{3}{|l|}{ Propiedades eléctricas } \\
\hline Conductividad eléctrica & $\begin{array}{ll}5,75 \mathrm{e}-21 & - \\
5,22 \mathrm{e}-20 & \\
\end{array}$ & $\%$ IACS \\
\hline \multicolumn{3}{|c|}{ Absorción y permeabilidad } \\
\hline $\begin{array}{l}\text { Absorción de agua } \\
\text { ( } 24 \text { horas) }\end{array}$ & $* 0,2-0,45$ & $\%$ \\
\hline \multicolumn{3}{|l|}{ Durabilidad } \\
\hline Agua (dulce) & & Excelente \\
\hline Agua (salada) & & Excelente \\
\hline Ácidos débiles & & Excelente \\
\hline Alcalinos fuertes & & Excelente \\
\hline Ácidos fuertes & & Uso limitado \\
\hline Radiación UV & & Pobre \\
\hline
\end{tabular}

Fuente: [19]. 

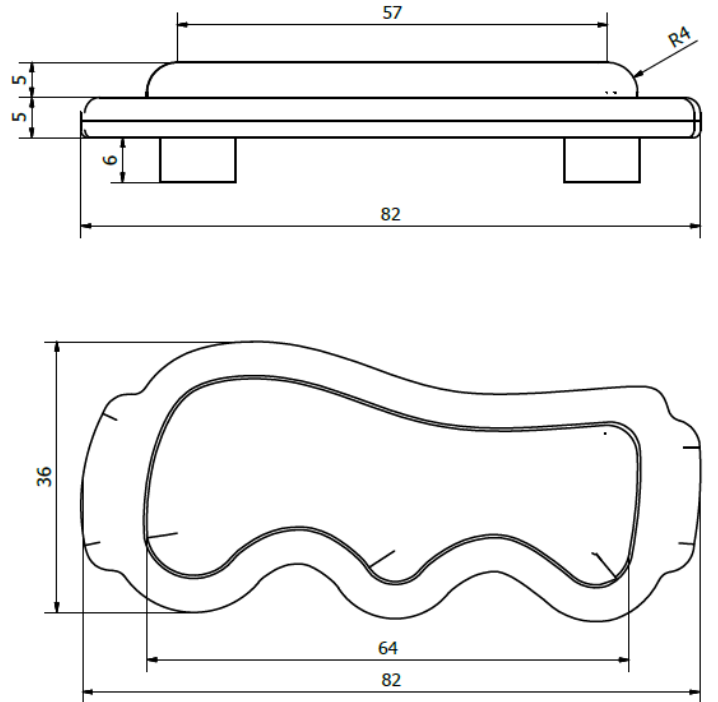

Figura 11. Planos dimensionales del dispositivo de relajación palmar. Fuente: elaboración propia.
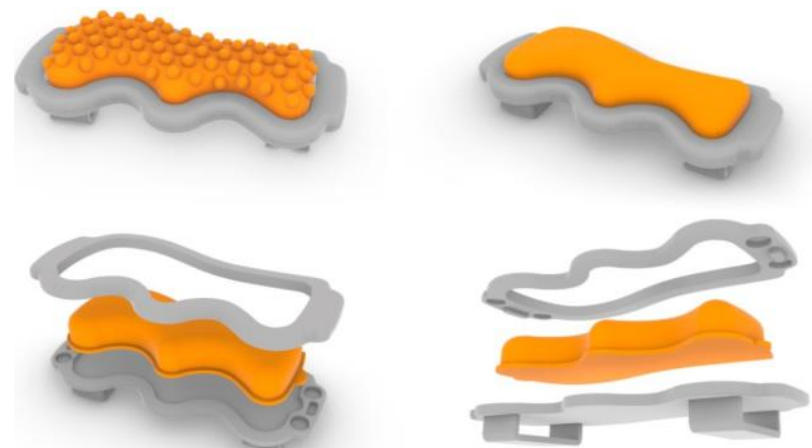

Figura 12. Modelo virtual del dispositivo de relajación palmar. Fuente: elaboración propia.
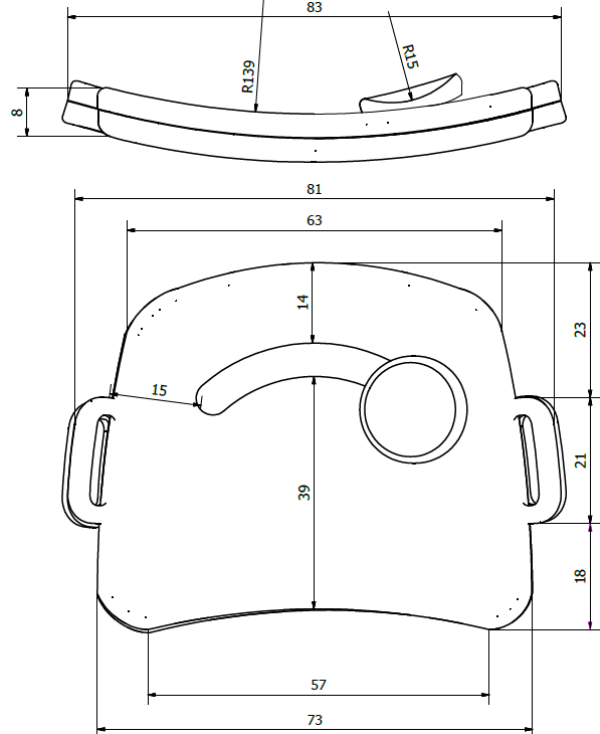

Figura 13. Planos dimensionales del dispositivo de movilidad pulgar. Fuente: elaboración propia.
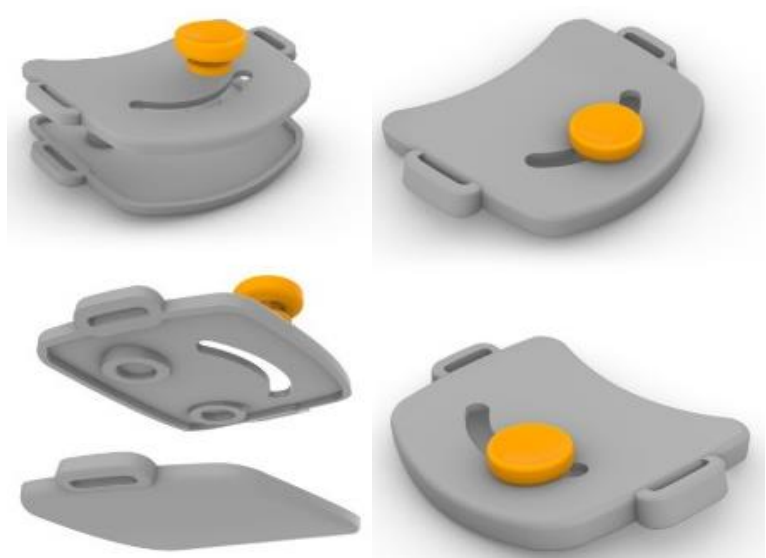

Figura 14. Modelo virtual del dispositivo de movilidad pulgar. Fuente: elaboración propia.

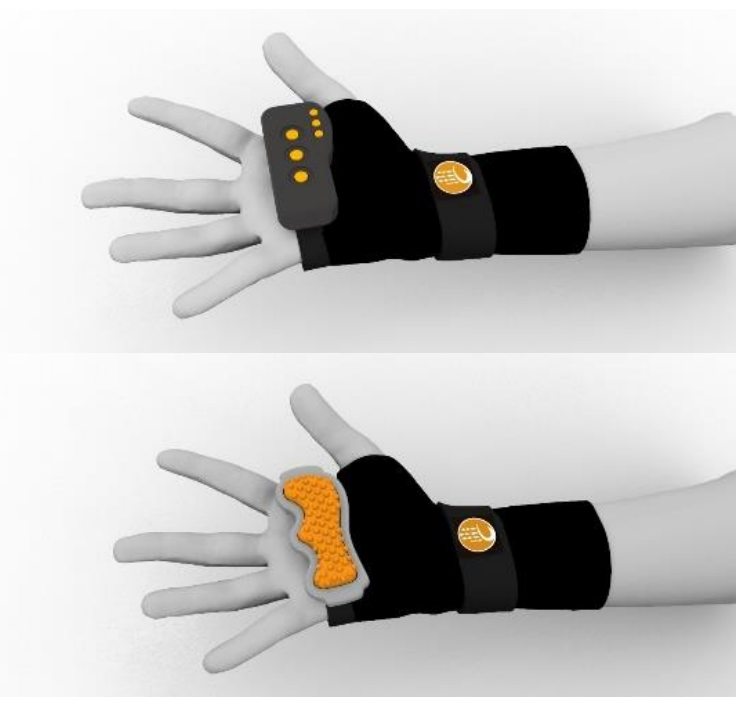

Figura 15. Vista en conjunto del instrumento terapéutico. Fuente: elaboración propia.

\subsection{Evaluación de usabilidad}

Se aplica una prueba para la evaluación de usabilidad con el propósito de validar la fase de diseño de detalle. Mediante esta herramienta se obtiene una retroalimentación sobre el instrumento de manera real. La prueba enfocada al usuario del instrumento tiene el propósito de valorar los puntos fuertes y débiles que existen en la interacción objeto-usuario, bajo condiciones reales. Está conformada por 22 preguntas que responden a 5 ámbitos (véase tabla 10), con una escala de valoración de 1 a 5 , donde 1 equivale a totalmente insatisfecho y 5 , a totalmente satisfecho. En esta evaluación participan un médico especialista y un paciente que sufre la patología. 


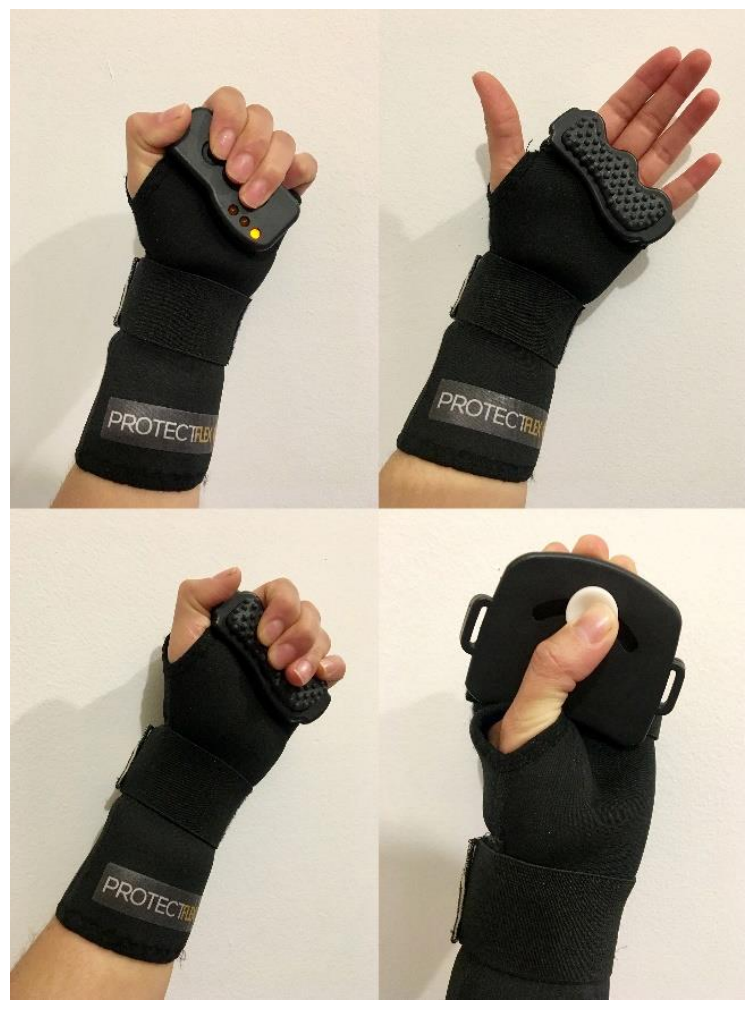

Figura 16. Prototipo del instrumento terapéutico. Fuente: elaboración propia.

Tabla 10. Ámbito de la prueba

\begin{tabular}{|l|l|}
\hline \multicolumn{1}{|c|}{ Ámbito } & \multicolumn{1}{c|}{ Descripción } \\
\hline $\begin{array}{l}\text { Comodidad } \\
\text { del guante }\end{array}$ & $\begin{array}{l}\text { Fácil colocación y uso de } \\
\text { instrumento. } \\
\text { Confiabilidad que brinda el } \\
\text { instrumento. }\end{array}$ \\
\hline $\begin{array}{l}\text { Ajuste a la } \\
\text { muñeca }\end{array}$ & Compresión adecuada en muñeca. \\
\hline $\begin{array}{l}\text { Ejercicios } \\
\text { para } \\
\text { deslizamiento } \\
\text { de tendones }\end{array}$ & $\begin{array}{l}\text { Movimientos de } \\
\text { - flexión y extensión de dedos en } \\
\text { forma grupal e individual; }\end{array}$ \\
\hline $\begin{array}{l}\text { Utilidad del del pulgar; } \\
\text { instrumento gancho, completo y } \\
\text { para } \\
\text { rehabilo. }\end{array}$ & $\begin{array}{l}\text { Estimulación de yema de dedos, } \\
\text { músculos y tendones afectados. }\end{array}$ \\
\hline $\begin{array}{l}\text { Ejecución de } \\
\text { movimientos } \\
\text { intuitivos }\end{array}$ & $\begin{array}{l}\text { Comprensión fácil por parte del } \\
\text { usuario sobre funcionamiento del } \\
\text { instrumento. }\end{array}$ \\
\hline
\end{tabular}

Fuente: elaboración propia.
Aplicando la prueba, se obtienen resultados satisfactorios (véase figura 17) en cuanto a su funcionalidad, lo que explica que el instrumento cumple con las especificaciones terapéuticas impuestas en este trabajo, basadas en las necesidades del usuario.

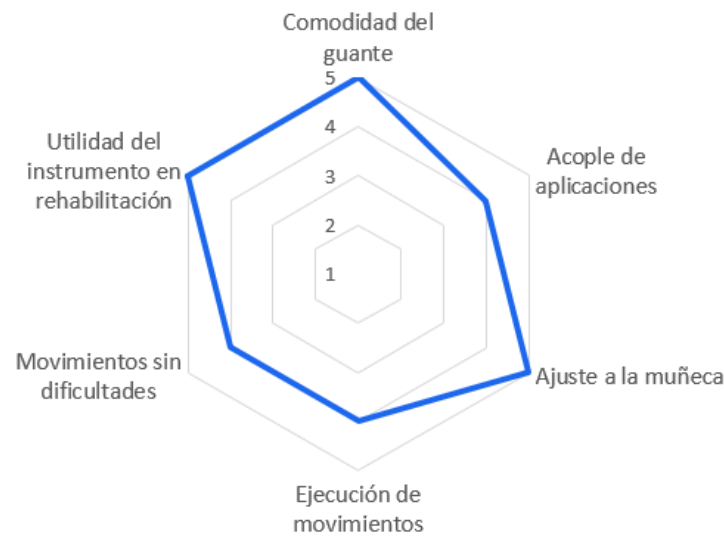

Figura 17. Resultados de prueba de usabilidad. Fuente: elaboración propia.

\section{Conclusiones}

Se desarrolló el diseño de un instrumento terapéutico para tratar la tenosinovitis De Quervain. Entre los síntomas causados por esta patología se encuentran entumecimiento, dolor y hormigueo, provocados por el desgaste en los tendones que conectan la muñeca y los dedos de la mano.

La tenosinovitis De Quervain se trata con rehabilitación terapéutica, por lo cual se utilizó el programa Tendon Gliding Exercise como base para el desarrollo del prototipo.

Se aplicó un proceso metodológico de diseño, iniciando con la planificación y aclaración de la tarea para obtener las necesidades de usuarios que posteriormente se convirtieron en requerimientos ingenieriles a cumplir en el instrumento.

Mediante el diseño conceptual, de realización y de detalle, se especificaron los aspectos funcionales, ergonómicos y estéticos del producto, obteniendo un instrumento de apoyo para terapias físicas. Dicho instrumento se caracteriza por poseer dispositivos intercambiables que motivan al usuario a realizar los movimientos ideales de flexión y extensión de los dedos, mismos que ayudan a liberar tensión en las articulaciones y tendones; de esta manera, se consigue la relajación de la mano y muñeca. 
Establecido el diseño se definen materiales, sistemas de funcionamiento, y se comprueba el cumplimiento de especificaciones del implemento terapéutico, mediante test de usabilidad, con resultados favorables en los ámbitos analizados.

\section{Referencias}

[1] M. Garrafa Núñez, M. C. García Martín, G. Sánchez Lemus, "Factores de riesgo laboral para tenosinovitis del miembro superior," Medicina y Seguridad Del Trabajo, vol. 61, no. 241, pp. 486-503, 2015.

[2] S. Gallagher, M. C. Schall Jr, "Musculoskeletal disorders as a fatigue failure process: evidence, implications and research needs," Ergonomics, vol. 60, no. 2, pp. 255-269, 2017, doi: 10.1080/00140139.2016.1208848.

[3] Organización Mundial de la Salud. Trastornos musculoesqueléticos. [En línea]. Disponible en: https://www.who.int/es/news-

room/factsheets/detail/musculoskeletal-conditions.

[4] S. L. James et al., "Global, regional, and national incidence, prevalence, and years lived with disability for 354 diseases and injuries for 195 countries and territories, 1990-2017: a systematic analysis for the Global Burden of Disease Study 2017," Lancet, vol. 392, no. 10159, pp. 1789-1858, 2018, doi: 10.1016/S0140-6736(18)32279-7

[5] D. Van Eerd et al., "Effectiveness of workplace interventions in the prevention of upper extremity musculoskeletal disorders and symptoms: an update of the evidence," Occup. Environ. Med., vol. 73, no. 1, pp. 62-70, 2016, doi: 10.1136/oemed-2015-102992.

[6] M. Van Middelkoop et al., "Effectiveness of interventions of specific complaints of the arm, neck, or shoulder (CANS): musculoskeletal disorders of the hand," Clin. J. Pain, vol. 25, no. 6, pp. 537-552, 2009, doi: 10.1097/AJP.0b013e31819ff52c.

[7] A. Jurado, I. Medina-Porqueres, Tendón: Valoración Y Tratamiento En Fisioterapia. 2008.

[8] E. Abril Belchí, J. Martínez Cañadas, "Interés del tratamiento osteopático en la enfermedad de De Quervain. A propósito de un caso," Fisioterapia, vol. 30, no. 6, pp. 299-304, 2008, doi: 10.1016/j.ft.2008.09.016.
[9] X. Gu et al., "Tendoscopic versus open release for de Quervain's disease: earlier recovery with 7.21-year follow-up," Journal of Orthopaedic Surgery and Research, vol. 14, no. 1, pp. 357, 2019, doi: 10.1186/s13018-019-1393-5.

[10] X. Miguéns, J. Formigo, Abordaje Médico Del Dolor En Rehabilitación. España: Enfoque Editorial SC, 2017.

[11] M. De la Iglesia, "Ejercicios Excéntricos En Patología Musculoesquelética," trabajo de fin de grado, Universidad Complutense, 2018.

[12] Á Sánchez-Cabeza, J. L. Arana-Echavarría, "Bases científicas para el diseño de un programa de ejercicios para la mano del paciente con artritis reumatoide," [En línea]. Disponible en: http://Sermef-Ejercicios.Org.

[13] W. Mosquera, G. Toapanta, "Dispositivo mecatrónico para rehabilitación pasiva de muñeca", trabajo de fin de grado, Universidad Técnica del Norte, 2017.

[14] G. Pahl, W. Beitz, J. Feldhusen, K.-H. Grote, Engineering Design. London: Springer London, 2007.

[15] K. Ulrich, S. Eppinger, Eds., Diseño Y Desarrollo De Productos. (5ta ed.) México: McGraw-Hill, 2013.

[16] B. Redwood, F. Schffer, B. Garret, The 3D Printing Handbook: Technologies, Design and Applications. 3D Hubs, 2017.

[17] M. A. Wehbé, "Tendon Gliding Exercises," Am. J. Occup. Ther., vol. 41, (3), pp. 164-167, 1987, doi: 10.5014/ajot.41.3.164.

[18] J. Panero, M. Zelnik, Las Dimensiones Humanas En Espacios Interiores. (7ma ed.) México: Ediciones G. Gili, S.A de C.V, 1996.

[19] R. W. Neu, V. Martinez, K. Kern, A. Lowe, Granta Desing Limited, "A unique teaching resource for materials”, 2019. [En línea]. Disponible en: https://www.grantadesign.com/wpcontent/uploads/2019/02/CES-EduPack-2019-ProductOverview.pdf. 